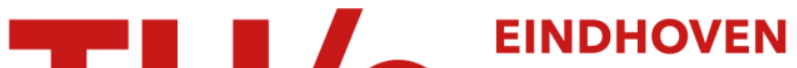 UNIVERSITY OF TECHNOLOGY
}

\section{An optimal estimation method for nonlinear models of mechanical systems}

Citation for published version (APA):

Molengraft, van de, M. J. G., Veldpaus, F. E., \& Kok, J. J. (1994). An optimal estimation method for nonlinear models of mechanical systems. Journal of Dynamic Systems, Measurement and Control : Transactions of the ASME, 116(4), 805-810. https://doi.org/10.1115/1.2899284

DOI:

$10.1115 / 1.2899284$

Document status and date:

Published: 01/01/1994

\section{Document Version:}

Publisher's PDF, also known as Version of Record (includes final page, issue and volume numbers)

\section{Please check the document version of this publication:}

- A submitted manuscript is the version of the article upon submission and before peer-review. There can be important differences between the submitted version and the official published version of record. People interested in the research are advised to contact the author for the final version of the publication, or visit the $\mathrm{DOI}$ to the publisher's website.

- The final author version and the galley proof are versions of the publication after peer review.

- The final published version features the final layout of the paper including the volume, issue and page numbers.

Link to publication

\section{General rights}

Copyright and moral rights for the publications made accessible in the public portal are retained by the authors and/or other copyright owners and it is a condition of accessing publications that users recognise and abide by the legal requirements associated with these rights.

- Users may download and print one copy of any publication from the public portal for the purpose of private study or research.

- You may not further distribute the material or use it for any profit-making activity or commercial gain

- You may freely distribute the URL identifying the publication in the public portal.

If the publication is distributed under the terms of Article 25fa of the Dutch Copyright Act, indicated by the "Taverne" license above, please follow below link for the End User Agreement:

www.tue.nl/taverne

Take down policy

If you believe that this document breaches copyright please contact us at:

openaccess@tue.nl

providing details and we will investigate your claim. 
2) Remark 3 is unnecessary because the stability criterion in this case will have a range of frequency between 0 and $2 \pi$.

\section{Concluding Remarks}

A numerical approach to model-matching controller synthesis has been introduced. The method is applicable when the system is open-loop stable and redundantly actuated. The major merits of the method are as follows.

1) The closed-loop system equation may be arbitrarily assigned.

2) Explicit knowledge of an open-loop model is not needed for the controller synthesis.

3) The workability of the designed controller may be verified during the synthesis process, even in the absence of an open-loop system model.

Admittedly, the need for two input channels is certainly a disadvantage for the method. However, this disadvantage is balanced to some extent by the fact that the minimum phase requirement in the open-loop system is lifted in cases where redundant inputs are available.

\section{References}

Åström, K. J., and Wittenmark, B., 1989, Adaptive Control, Addison Wesley Chen, C. T., 1984, Linear System Theory and Design. Holt, Rinehart, and Winston, New York.

Chotai, A., and Owens, D. H., et al., 1984, "Design of Smith Control Schemes for Time-Delay Systems Based on Plant Step Data," Int. J. Control, Vol. 40, No. 2.

Ljung, L., 1987, System Identification-Theory for the User, Prentice Hall, Englewood Cliffs, N.J.

McLean, D., 1990, Automatic Flight Control Systems, Prentice Hall International, New York.

Owens, D. H., 1980, "Large-Scale Systems Analysis Using Approximate Inverse Models," IEEE Trans. on Auto. Contr., pp. 328-330.

Owens, D. H., and Chotai, A., 1984, "Robust Sampled Regulators for Stable Systems from Plant Step Data," Automatica, Vol. 20, pp. 465-469.

Rogers, E., and Owens, D. H., 1990, "Simulation-Based Stability Tests for Differential Unit Memory Linear Multipass Processes," Int. J. Control, Vol. 51

Wolovich, W. A., 1974, Linear Multivariable Systems, Springer-Verlag, Berlin.

\section{A P P E N D I X}

The Existence of $R_{1}(s)$ and $R_{2}(s)$

Because $G(s)=\mathfrak{g}(s) \mathscr{G}(s)$, (4) implies that

$$
B_{1}(s) R_{1}(s)+B_{2}(s) R_{2}(s)=A(s) \mathcal{G}(k) .
$$

To show that the pair $R_{1}(s)$ and $R_{2}(s)$ always exists for any arbitrary $\mathcal{S}(s)$, express $B_{1}(s)$ and $B_{2}(s)$ in the following form

$$
B_{1}(s)=\sum_{i=0}^{\bar{n}} b_{1, i} s^{\bar{n}-i} \quad \text { and } \quad B_{2}(s)=\sum_{i=0}^{\bar{n}} b_{2, i} s^{\bar{n}-i}
$$

and form the $(m+\bar{n}+1) \times 2(m+1)$ matrix

$$
\mathscr{B}_{m}=\left[\begin{array}{cccccc}
b_{1,0} & & & b_{2,0} & & \\
\vdots & \ddots & & \vdots & \ddots & \\
b_{1, \bar{n}} & & b_{1,0} & b_{2, \bar{n}} & & b_{2,0} \\
& \ddots & \vdots & & \ddots & \vdots \\
& & b_{1, \bar{n}} & & & b_{2, \bar{n}}
\end{array}\right] .
$$

If $\mathscr{B}_{m}$ is column singular, then it is always possible to satisfy $B_{1}(s) R_{1}(s)+B_{2}(s) R_{2}(s)=0$ with some nonzero $R_{i}(s)$. However, for this equation to hold true requires that $\frac{R_{1}(s)}{R_{2}(s)}=$
$-\frac{B_{2}(s)}{B_{1}(s)}$. Given a coprime $B_{i}(s)$ pair, this is possible only if $m \geq \bar{n}$.

Since $\mathscr{B}_{m}$ is square when $m=\bar{n}-1$, a full rank $\mathscr{B}_{\bar{n}-1}$ is guaranteed. And for any $m>\bar{n}-1$, an additional $2(m-\bar{n}$ +1 ) columns will be added on to $\mathfrak{B}_{m}$. At the same time, $m$ $-\bar{n}+1$ linearly independent rows will also be introduced into the matrix. Thus, the full rank of $\mathscr{B}_{m}$ will be maintained for all $m \geq \bar{n}-1$ and the arbitrary placement of $B_{1}(s) R_{1}(s)$ $+B_{1}(s) R_{1}(s)$ is ensured.

Two final comments should be made. First, if desired $\Theta(s)$ happens to be inside the column space of $\mathscr{B}_{m}$, (A.1) can also be satisfied for $m<\bar{n}-1$. In general, then, $m \geq \bar{n}-1$ is a sufficient but not a necessary conditions for (A.1) to be satisfied. Second, the lowest possible value of $m$ in (A.1) will occur when $\mathscr{S}(s)=1$ and thus when $m=n-\bar{n}$. In general, then, it is necessary that $m \geq \max (n-\bar{n}, \bar{n}-1)$.

\section{An Optimal Estimation Method for Nonlinear Models of Mechanical Systems}

\section{J. G. van de Molengraft, ${ }^{1}$ F. E. Veldpaus, ${ }^{1}$ and J. J. Kok ${ }^{1}$}

This paper presents an optimal estimation method for nonlinear mechanical systems. The a priori knowledge of the system in the form of a nonlinear model structure is taken as a starting point. The method determines estimates of the parameters and estimates of the positions, velocities, accelerations, and inputs of the system. The optimal estimation method is applied to an experimental mechanical system. The unknown parameters in this system relate to inertia, friction and elastic deformation. It is shown that the optimal estimation method on the basis of a relatively simple model structure can lead to a useful description of the system.

\section{Introduction}

The subject of this paper is the development of an estimation method for non-linear mechanical systems. Attention will be focused on mechanical systems that have to be controlled to obtain the system behavior desired, such as manipulators. Control design requires a mathematical model of the system to take into account the system dynamics.

For mechanical systems an adequate model structure can usually be derived by theoretical modeling: simplifying assumptions are made and physical laws are applied. A model

\footnotetext{
${ }^{1}$ WFW, Control and Identification of Mechanical Systems, Department of Mechanical Engineering, Eindhoven University of Technology, 5600 MB Eindhoven, The Netherlands.

Contributed by the Dynamic Systems and Control Division of The American Society of Mechanical Engineers. Manuscript received by the DSCD July 21, 1993; revised manuscript received September 18, 1992. Associate Technical Editor: S. Jayasuriya.
} 
structure thus obtained contains a number of variables and parameters. Variables are positions, velocities, accelerations and control inputs acting on the system, such as forces and torques. Parameters are physical constants such as mass, stiffness etc. The variables and parameters have to be determined by estimation, i.e., the confrontation between measurements on the system and the theoretical model. Measurements can be any combination of measured variables and/ or measured parameters. Because of the physical relevance of the model structure, the estimated model may be expected to be more or less generally valid, i.e., to provide an adequate description of the system behavior for different input signals. For this reason, this model structure will be taken as a starting point for further considerations.

The theory presented in this paper shows a number of differences with most methods in literature.

In literature the difference between model and system is often considered as a stochastic model uncertainty (e.g., Eykhoff [1]). However, for mechanical systems the assumption of stochastic model uncertainty is mostly not founded. During the modeling stage simplifications are made which usually lead to a mainly deterministic difference between system and model. The quality of the estimated model can be judged by making a residual analysis and finally by looking at the obtained control result.

Two approaches for nonlinear models are often found in literature: linearization and transformation of the nonlinear model into a linear one. The idea is that, once the problem has been translated into a linear one, all available estimation methods for linear models can be used. Linearizing the model, however, always implies neglecting higher-order terms and may fail when strong non-linearities are involved. Transformation has been successfully applied in control practice (Isidori [2]), but has turned out to be a complicated matter in estimation because of the strong restrictions imposed on the model in question (e.g., Misawa and Hedrick [3]). For this reason, this paper aims at solving the estimation problem using the non-linear model itself.

Estimation of variables and estimation of parameters are usually treated as separate problems. In general, however, model variables as well as model parameters will be unknown. The estimation method discussed in this paper, therefore, provides estimates for all model quantities.

A specific feature of the theory in this paper is the combination of the above aspects within one theoretical framework.

In Section 2 of this paper a general model structure for mechanical systems will be discussed. In Section 3 the estimation problem will be further elaborated. A numerical solution will be given. In Section 4 the theory will be applied to a non-linear mechanical system. Section 5 will show the conclusions.

\section{The Estimation Model}

2.1 The Dynamic Model. Generally the mechanical systems in question can be descibed by using a mathematical model of the following form (Heeren [4], Paul [5]):

$$
\begin{gathered}
\dot{\mathbf{s}}=\mathbf{v} ; \dot{\mathbf{v}}=\mathbf{a} ; \dot{\boldsymbol{\theta}}=\mathbf{0} \\
M(\mathbf{s}, \boldsymbol{\theta}, t) \mathbf{a}+\mathbf{h}(\mathbf{s}, \mathbf{v}, \boldsymbol{\theta}, t)+H(\mathbf{s}, \mathbf{v}, \boldsymbol{\theta}, t) \mathbf{u}=\mathbf{0} \\
\mathbf{y}=\mathbf{b}(\mathbf{s}, \mathbf{v}, \boldsymbol{\theta}, \mathbf{u}, \mathbf{a}, t)
\end{gathered}
$$

Here, $\mathbf{s}$ is the $n$ column with degrees of freedom (positions), $\mathbf{v}$ is the $n$ column with the first time derivative of $\mathbf{s}$ (velocities), $\mathbf{a}$ is the $n$ column with the first time derivative of $\mathbf{v}$ (accelerations) and $\boldsymbol{\theta}$ is the $p$ column with unknown, constant parameters. At the modeling stage, the model parameters $\boldsymbol{\theta}$ represent physical system properties, such as mass and stiffness. Column $\mathbf{u}$ is the $m$ column with adjustable system inputs to control the system. As inputs, quantities will be chosen that can directly be associated with degrees of freedom, such as actuator forces. Because of this, the equation of motion (2) can be assumed to be linear in $\mathbf{u}$. In Eq. (2), $M$ is the $n \times n$ mass matrix, $\mathbf{h}$ is the $n$ column containing nonlinear terms, such as Coriolis and centrifugal forces, the effect of gravity and damping, and $H$ is the $n \times m$ distribution matrix. In Eq. (3), $\mathbf{y}$ is the $q$ column with controlled outputs defined by $q$ column $\mathbf{b}$ with nonlinear functions of the model variables and the independent time variable $t / t \in\left[t_{0}, t_{e}\right]$. Appropriate initial conditions for Eq. (1) are assumed. Eq. (2) is, in general, strongly nonlinear in the model variables and often linear in the parameters. However, from the estimation point of view the degree of nonlinearity of Eq. (2) is increased by the presence of combined terms of variables and parameters.

2.2 The Measurement Model. The model should accurately predict the outputs $\mathbf{y}$ resulting from the inputs $\mathbf{u}$. In most cases, column $\mathbf{y}$ should track a desired trajectory in space (control objective). It seems obvious, therefore, that the inputs and outputs of the system should be measured for the purpose of the estimation. The inputs can usually be measured without difficulty, but measuring the controlled outputs is not always feasible. For instance, problems may arise when the end-effector position of a manipulator is measured. In addition, the a priori knowledge in the form of Eqs. (1) and (2) should be used. In general, a unique estimation of the model quantities will require other measurements besides those of the inputs and outputs. This comes under the observability problem, a mathematical treatment of which can be found, for instance, in Walter [6] and Tunati and Tarn [7]. The quantities playing a role in the model, including the inputs and outputs of the system, should, as far as possible, be measured. A model that fits the measurements well is expected to describe the inputoutput relation accurately, if the measurrements are taken under circumstances similar to the final control situation.

In general, knowledge of the measured quantities will result in a model of the following form:

$$
\mathbf{z}=\mathbf{g}(\mathbf{s}, \mathbf{v}, \boldsymbol{\theta}, t)+G(\mathbf{s}, \mathbf{v}, \boldsymbol{\theta}, t) \mathbf{a}+R(\mathbf{s}, \mathbf{v}, \boldsymbol{\theta}, t) \mathbf{u}
$$

where $\mathbf{z}$ is the $l$ column with model measurements, $\mathbf{g}$ is an $l$ column, $G$ is an $l \times n$ matrix and $R$ is an $l \times m$ matrix. The controlled outputs $y$ are not necessarily part of the model measurements $\mathbf{z}$. Equation (4) does not correspond to the standard form in literature, due to the presence of accelerations $\mathbf{a}$. They could be eliminated by using the equation of 
motion (2). However, in this paper accelerations are being handled directly, just like the other variables.

The combination of Eqs. (1) and (2) and Eq. (4) is called the system model. The system model is assumed to be linear in $\mathbf{u}$. This assumption, which, in general, does not play a restrictive role for the systems in question, substantially simplifies the mathematical elaboration.

2.3 Estimation. The objective is to find values of $\theta$ for which the system model best describes the measurement data. From Eqs. (1), (2) and (4) it is evident that the choice of $\boldsymbol{\theta}$ cannot be seen separate from the choice of $\mathbf{s}\left(t_{0}\right), \mathbf{v}\left(t_{0}\right)$ and $\mathbf{u}$. These quantities have to be estimated, too. In general, it is impossible to choose quantities $\boldsymbol{\theta} \mathbf{s}\left(t_{0}\right), \mathbf{v}\left(t_{0}\right)$ and $\mathbf{u}$ that result in perfect match between the model quantity $\mathbf{z}$ and the measurements $\mathbf{z}_{m}$. The model and the measurement data, therefore, will be made to coincide as far as possible. For that purpose the estimation model is introduced:

$$
\begin{gathered}
\dot{\hat{\mathbf{s}}}=\hat{\mathbf{v}}+\boldsymbol{\xi}_{1} ; \dot{\hat{\mathbf{v}}}=\hat{\mathbf{a}}+\boldsymbol{\xi}_{2} ; \dot{\hat{\boldsymbol{\theta}}}=\boldsymbol{\xi}_{3} \\
M(\hat{\mathbf{s}}, \hat{\boldsymbol{\theta}}, t) \hat{\mathbf{a}}+\mathbf{h}(\hat{\mathbf{s}}, \hat{\mathbf{v}}, \hat{\boldsymbol{\theta}}, t)+H(\hat{\mathbf{s}}, \hat{\mathbf{v}}, \hat{\boldsymbol{\theta}}, t) \hat{\mathbf{u}}=\zeta_{1} \\
\hat{\mathbf{z}}=\mathbf{g}(\hat{\mathbf{s}}, \hat{\mathbf{v}}, \hat{\boldsymbol{\theta}}, t)+G(\hat{\mathbf{s}}, \hat{\mathbf{v}}, \hat{\boldsymbol{\theta}}, t) \hat{\mathbf{a}} \\
+R(\hat{\mathbf{s}}, \hat{\mathbf{v}}, \hat{\boldsymbol{\theta}}, t) \hat{\mathbf{u}} ; \mathbf{z}_{m}=\hat{\mathbf{z}}+\zeta_{2}
\end{gathered}
$$

where $\hat{\mathbf{s}}, \hat{\mathbf{v}}, \hat{\boldsymbol{\theta}}, \hat{\mathbf{u}}$ and $\hat{\mathbf{a}}$ are estimators of $\mathbf{s}, \mathbf{v}, \boldsymbol{\theta}, \mathbf{u}$ and $\mathbf{a}$, $\hat{\mathbf{z}}$ is an estimator of the measured quantities $\mathbf{z}$ and $\boldsymbol{\xi}_{1}, \boldsymbol{\xi}_{2}, \boldsymbol{\xi}_{3}$, $\boldsymbol{\zeta}_{1}$ and $\boldsymbol{\zeta}_{2}$ are residuals. Estimates $\hat{\mathbf{s}}, \hat{\mathbf{v}}, \hat{\boldsymbol{\theta}}$, $\hat{\mathbf{u}}$ and $\hat{\mathbf{a}}$ should be such that the smallest possible residuals are obtained to ensure that the measurements are optimally explained by the model.

The estimation model can be formulated more compactly. For this purpose, the augmented state estimator $\hat{\mathbf{x}}$ and the augmented input estimator $\hat{\mathbf{p}}$ are defined as $\hat{\mathbf{x}}^{T}=[\hat{\mathbf{s}} \hat{\mathbf{v}} \hat{\boldsymbol{\theta}}]$ and $\hat{\mathbf{p}}^{T}=[\hat{\mathbf{u}} \mathbf{a}]$. Now, it is clear that Eq. (5) is equivalent to:

$$
\begin{aligned}
\dot{\mathbf{x}}=A \hat{\mathbf{x}}+B \hat{\mathbf{p}}+\xi ; A=\left[\begin{array}{lll}
O & I & O \\
O & O & O \\
O & O & O
\end{array}\right] ; \\
B=\left[\begin{array}{ll}
O & O \\
O & I \\
O & O
\end{array}\right] ; \boldsymbol{\xi}=\left[\begin{array}{l}
\boldsymbol{\xi}_{1} \\
\boldsymbol{\xi}_{2} \\
\xi_{3}
\end{array}\right]
\end{aligned}
$$

Furthermore, Eqs. (6) and (7) can be formulated as:

$$
\begin{aligned}
E \mathbf{z}_{m} & =\mathbf{f}(\hat{\mathbf{x}}, t)+F(\hat{\mathbf{x}}, t) \hat{\mathbf{p}}+\boldsymbol{\xi} \\
E & =\left[\begin{array}{l}
O \\
I
\end{array}\right] \mathbf{f}(\hat{\mathbf{x}}, t)=\left[\begin{array}{l}
\mathbf{h}(\hat{\mathbf{x}}, t) \\
\mathbf{g}(\hat{\mathbf{x}}, t)
\end{array}\right] \\
F(\hat{\mathbf{x}}, t) & =\left[\begin{array}{ll}
H(\hat{\mathbf{x}}, t) & M(\hat{\mathbf{x}}, t) \\
R(\hat{\mathbf{x}}, t) & G(\hat{\mathbf{x}}, t)
\end{array}\right] \\
\zeta & =\left[\begin{array}{l}
\zeta_{1} \\
\zeta_{2}
\end{array}\right]
\end{aligned}
$$

The problem now is to determine quantities $\hat{\mathbf{x}}$ and $\hat{\mathbf{p}}$ that minimize $\xi$ and $\zeta$.

\section{Solution to the Estimation Problem}

3.1 Optimal Estimation. The estimation model (8), (9) is taken as a starting point. The aim is to find estimates $\hat{\mathbf{x}}$ and $\hat{\mathbf{p}}$ for which the residuals are as small as possible.
This can be mathematically formulated as follows: find $\hat{\mathbf{x}}(\tau)$ and $\hat{\mathbf{p}}(\tau) \mathrm{i} \tau \in\left[t_{0}, t\right]$ for which cost functional

$$
\begin{array}{r}
J[\hat{\mathbf{x}}, \hat{\mathbf{p}}]=\int_{t_{0}}^{t}\left\{\frac{1}{2} \xi^{T}(\tau) W(\tau) \xi(\tau)+\frac{1}{2} \zeta^{T}(\tau) V(\tau) \zeta(\tau)\right\} d \tau+ \\
+\frac{1}{2}\left[\hat{\mathbf{x}}\left(t_{0}\right)-\mathbf{q}_{0}\right]^{T} R_{0}\left[\hat{\mathbf{x}}\left(t_{0}\right)-\mathbf{q}_{0}\right]
\end{array}
$$

is minimized under the constraint Eqs. (8) and (9). Here, $W(\tau)$ is a positive definite weighting matrix expressing confidence in Eq. (8), $V(\tau)$ is a semi-positive definite weighting matrix expressing confidence in Eq. (9), $\mathbf{q}_{0}$ is a column representing initial knowledge about $\mathbf{x}\left(t_{0}\right)$ and $R_{0}$ is a positive definite weighting matrix expressing confidence in $\mathbf{q}_{0}$. The weighting matrices have to be non-negative definite to guarantee the existence of a minimum. Matrix $W$ must be positive definite, otherwise the physical coupling of the state estimates according to Eq. (1) would be lost and a unique solution would no longer be guaranteed. Matrix $V$ may be semi-positive definite as long as matrix $F^{T} V F$ is regular. The weighting matrices can be chosen symmetric without loss of generality. In most cases they will be chosen diagonal.

A necessary, but not sufficient condition for $J$ to be minimal is $\delta J=0 \forall \delta \hat{\mathbf{x}}, \delta \hat{\mathbf{p}}$. Assuming that the function $\mathbf{f}$ and $F$ are differentiable at least once, the following results are obtained:

$$
\begin{gathered}
\dot{\hat{\mathbf{x}}}=A \hat{\mathbf{x}}+B \hat{\mathbf{p}}+W^{-1} \boldsymbol{\lambda} \\
\dot{\boldsymbol{\lambda}}=-A^{T} \boldsymbol{\lambda}-C^{T} V[E \mathbf{z}-\mathbf{f}-F \hat{\mathbf{p}}] ; C=\frac{\partial \mathbf{f}}{\partial \hat{\mathbf{x}}}+\frac{\partial F}{\partial \hat{\mathbf{x}}} \hat{\mathbf{p}} \\
\hat{\mathbf{p}}=\left(F^{T} V F\right)^{-1}\left[\mathrm{~B}^{T} \boldsymbol{\lambda}+F^{T} V(E \mathbf{z}-\mathbf{f})\right]
\end{gathered}
$$

with boundary conditions $\boldsymbol{\lambda}\left(t_{0}\right)=R_{0}\left[\hat{\mathbf{x}}\left(t_{0}\right)-\mathbf{q}_{0}\right]$ and $\boldsymbol{\lambda}(t)=$ 0 . Column $\boldsymbol{\lambda}$ contains the so-called Lagrange multipliers or co-states. For simple notation, the function arguments have been omitted. Equations (11) to (13) hold for all $\tau \mid \tau \in\left[t_{0}\right.$, $t$ ]. The relation between $\boldsymbol{\xi}$ and $\boldsymbol{\lambda}$ is shown by comparing Eq. (8) and Eq. (11).

Solving this Two-Point Boundary Value Problem (TPBVP) yields smoothed estimates $\hat{\mathbf{x}}(\tau)$ and $\hat{\mathbf{p}}(\tau)$. The Jacobian of the differential Eqs. (11) and (12) is a Hamilton matrix. Consequently, numerical integration of Eqs. (11) and (12) in the same direction is unstable and standard shooting methods cannot be used (Jacobson and Mayne [8]). Various numerical methods have been proposed for nonlinear TPBVP's, such as difference methods, multiple shooting methods and forward-backward shooting methods. In this paper the first-order approximation as proposed in [9] is used.

\section{An Example}

4.1 The $x y$-Table. The system considered in this section is a so-called $x y$-table. A detailed description can be found in Heeren [4]. A schematic representation is given in Fig. 1. Two parallel slideways each support a slide. They are connected via belts to a torsion-flexible spindle, which is driven by motor 1 . A third slideway rests on the slides and supports the end-effector, which is belt-driven by motor 2 .

The aim is to find a model of the $x y$-table for control 


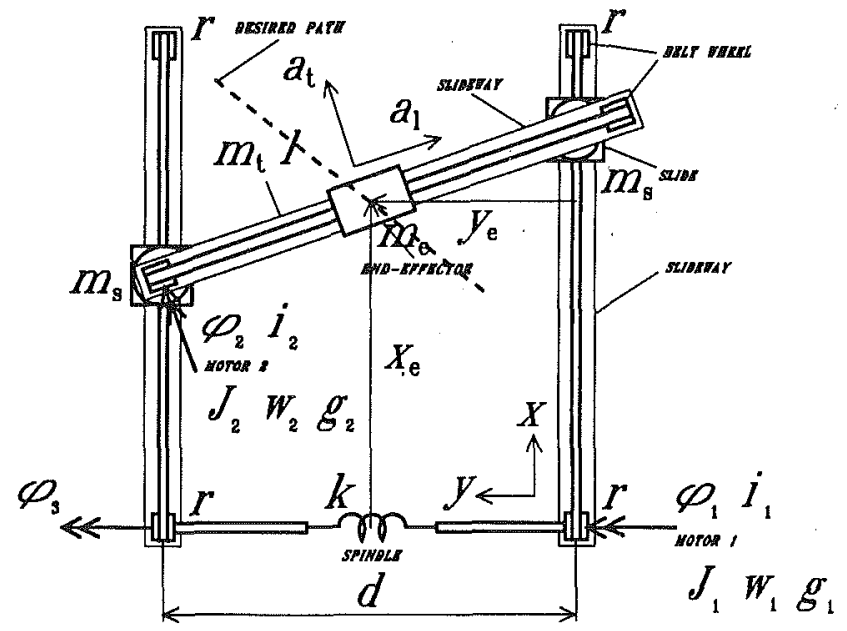

Fig. 1 The $x y$-table: model, measurements and desired path

$\varphi_{i} \quad \begin{aligned} & \text { angular position of motor } i, \\ & i=1,2\end{aligned}$
$\varphi_{3} \quad \begin{gathered}\text { angular position of spindle } \\ \text { end }\end{gathered}$
$i_{l} \quad$ current of motor $i$
$J_{i} \quad$ rotation inertia of motor $i$
$m_{s}$ mass of slide
$m_{t}$ mass of transverse slideway
$m_{0}$ mass of end-effector

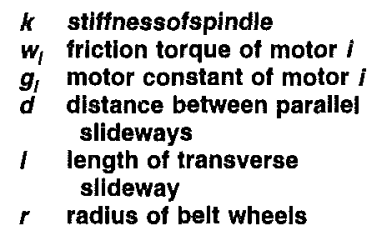

design. The position of the end-effector is defined as the controlled output and the motor currents as the control inputs. The motor currents should be such that the end-effector follows the path shown in Fig. 1.

4.2 The Model. The belts are assumed to be rigid. Furthermore, it is assumed that there is no play between the construction parts. The rotation inertia of the belt wheels is neglected. Friction in the system is assumed to occur mainly in the motors and to a lesser extent in the contact areas between belt and belt wheel and in the sliding contact areas. Only dry friction is modeled. It is assumed that there is a linear, static relation between the motor current and the generated torque.

The equation of motion for the above model is given by Eq. (2) where $\mathbf{s}^{T}=\left[\varphi_{1} \varphi_{2} \varphi_{3}\right]$ and $\mathbf{u}^{T}=\left[i_{1} i_{2}\right]$. The formula manipulation software package MAPLE (MAPLE Reference Manual [10]) is used to derive the equation of motion using the Lagrange equation. Terms in $\mathbf{h}$ are due to friction and to the deformation of the spindle. More details about $M, \mathbf{h}$ and $H$ are given in [9]. Parameters $J_{1}$ and $J_{2}$ are considered to be unknown, because they should also allow for all inertia in the vicinity of the motors (encoders, transmissions etc.). Friction forces $w_{1}$ and $w_{2}$ are difficult to measure locally and are unknown. Stiffness $k$ of the spindle is also regarded as an unknown parameter. So, the unknown parameters read $\boldsymbol{\theta}^{T}=\left[J_{1} J_{2} w_{1} w_{2} k\right]$. Column $\boldsymbol{\theta}$ will be determined by means of optimal estimation.

The desired path is approximately realized by using a simple PD feedback of the position and velocity errors of the motors. An experiment is performed in which the endeffector travels along the desired path six times forward and backward. The following quantities are measured as a function of time (see Fig. 1):

1. the position of the end-effector: $z_{1}=x_{e}$ and $z_{2}=y_{e}$,
2. the acceleration of the end-effector: $z_{3}=a_{t}$ and $z_{4}=a_{l}$,

3. the angular position of the motors: $z_{5}=\varphi_{1}$ and $z_{6}=$ $\varphi_{2}$ and

4. the motor currents: $z_{7}=i_{1}$ and $z_{8}=i_{2}$.

The position of the end-effector is measured by an optical system (Heeren ([4]). The measurements can be written in the form of Eq. (4). More details about $\mathbf{g}, G$, and $R$ are given in [9].

The experiment has been repeated several times. The differences between the realizations are very small, which emphasizes the deterministic nature of the system. The measuring equipment is accurate enough to be assumed ideal (Vincent [11]).

Yet, values must be chosen for column $\mathbf{q}_{0}$ and weighting matrices $W, V$, and $R_{0}$. The elements of $\mathbf{q}_{0}$ relating to positions and velocities are chosen equal to the desired values at $\tau=t_{0}$. The elements relating to inertia are taken from the motor specifications and the elements relating to friction in the system by means of simple experiments. An initial value for the unknown stiffness is calculated by using a standard formula for a steel torsion strip (linear material behavior). This results in: $\mathbf{q}_{0}^{T}=$

$\left[\begin{array}{ccccccccc}60 & 60 & 0 & 0 & 1.280 \cdot 10^{-3} & 0.600 \cdot 10^{-4} & 0.370 & 0.080 & 0.460 \\ \mathrm{rad} & \mathrm{rad} & \mathrm{rad} / \mathrm{s} & \mathrm{rad} / \mathrm{s} & \mathrm{kgm}^{2} & \mathrm{kgm}^{2} & \mathrm{Nm} & \mathrm{Nm} & \mathrm{Nm} / \mathrm{rad}\end{array}\right]$

As can be seen in $\mathbf{q}_{0}$, the model quantities strongly differ in magnitude. This complicates the choice of the weighting matrices. An attempt is made to choose the matrices in a more or less systematic way. First, the order of magnitude of the separate terms in the equations weighted is determined by looking at the measurement data and the initial parameter estimates. Next, the reciprocal value of the square of the order of magnitude is taken as a basic value for the corresponding weighting factor. Now, all terms in the functional are of the same order. Next, if necessary, relative differences between the weighting factors can be introduced. This strategy leads to the matrices given in [9]. Relatively heavy weight is attached to the measurements, because they represent the inputs and outputs of the system. Matrix $R_{0}$ is chosen small, especially with respect to the parameter estimates. So, the optimal smoother is free to choose its own initial estimates.

4.3 Results. Consider the time interval $\tau \in\left[t_{0}, t_{e}\right]$ where $t_{0}=3.33 \mathrm{~s}$ and $t_{e}=4.99 \mathrm{~s}$. This interval corresponds to one upward and one downward part of the trajectory. This-fairly arbitrary - choice is made to reduce the computational effort required for estimation, while it is expected that physically relevant parameter estimates obtained on this part of the trajectory will be suitable for the other parts, too.

The following parameter set is obtained:

$\hat{\boldsymbol{\theta}}^{T}=$

$$
\left[\begin{array}{ccccc}
2.099 \cdot 10^{-3} & 1.437 \cdot 10^{-4} & 0.468 & 0.151 & 0.245 \\
\mathrm{kgm}^{2} & \mathrm{kgm}^{2} & \mathrm{Nm} & \mathrm{Nm} & \mathrm{Nm} / \mathrm{rad}
\end{array}\right]
$$

Position estimates $\hat{\varphi}_{1}, \hat{\varphi}_{2}$, and $\hat{\varphi}_{3}$ are shown in Fig. 2. The difference between $\hat{\varphi}_{1}$ and $\hat{\varphi}_{3}$ represents the elastic deformation of the spindle. The residuals on the end-effector position measurements are shown in Fig. 3. They are the same order of magnitude as the elastic deformation. The same applies to 


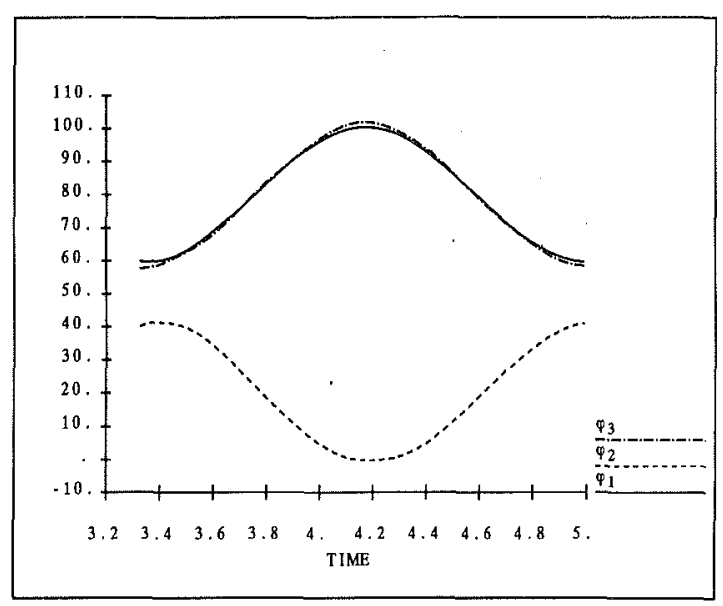

Fig. 2 Motor position estimates $\hat{\varphi}_{1}, \hat{\varphi}_{2}$ and $\hat{\varphi}_{3}$ (in rad)

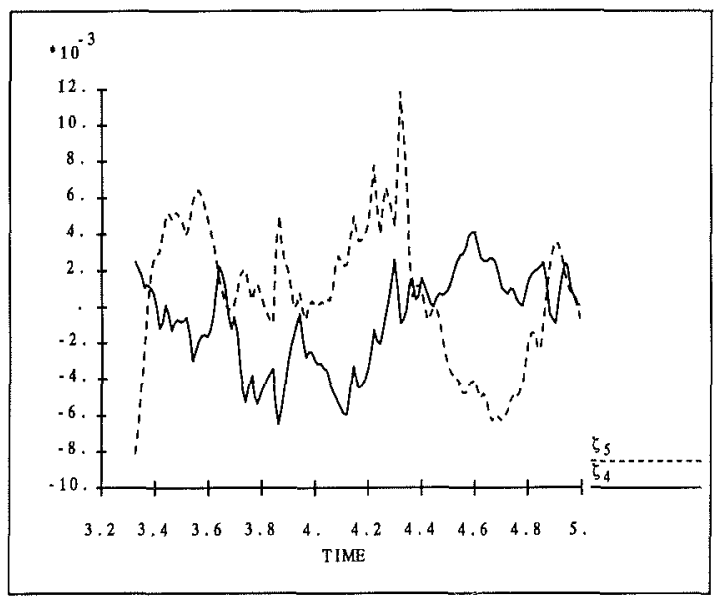

Fig. 3 Residuals $\zeta_{4}$ and $\zeta_{5}$ on the end-effector position measurements (in $m$ )

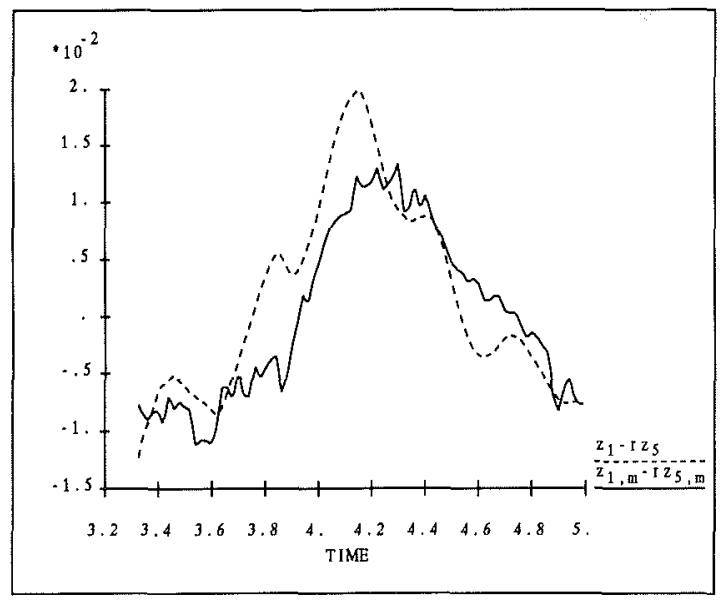

Fig. 4 Deformation in x-direction (in $m$ )

the motor position measurements. However, the deformation itself has been qualitatively well observed, as illustrated by Fig. 4, which shows the difference $x_{e}-\varphi_{1} r$, estimated as well as measured. The stiffness estimate, therefore, is expected to be physically relevant. Figure 4 also shows that the model

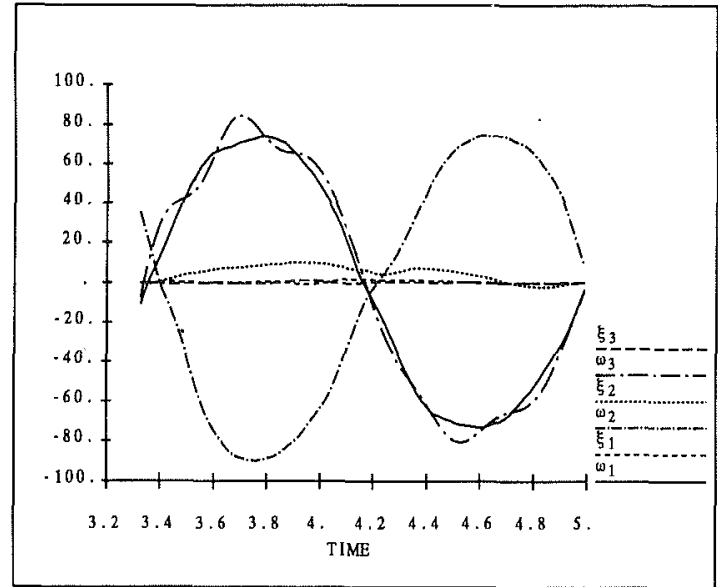

Fig. 5 Velocity estimates $\hat{\omega}_{1}, \hat{\omega}_{2}, \hat{\omega}_{3}$ and residuals $\xi_{1}, \xi_{2}, \xi_{3}$ (in rad/s)

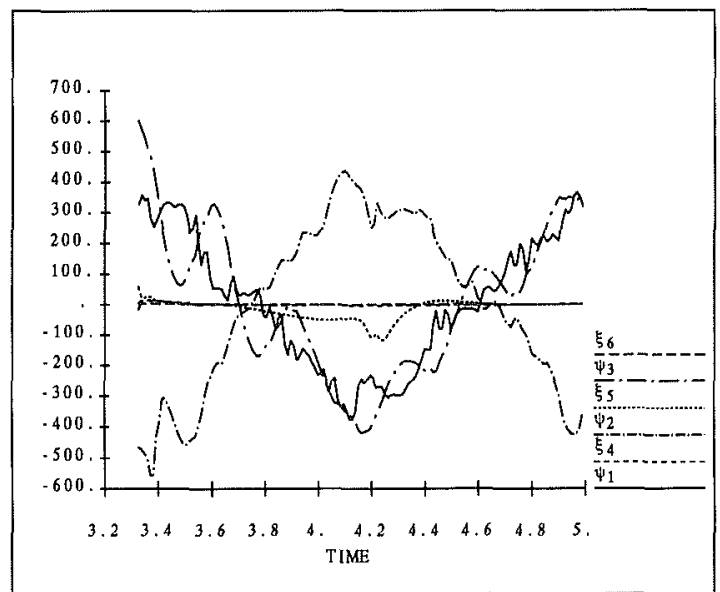

Fig. 6 Acceleration estimates $\hat{\psi}_{1}, \hat{\psi}_{2}, \hat{\psi}_{3}$ and residuals $\xi_{4}, \xi_{5}, \xi_{6}\left(\mathrm{in} \mathrm{rad} / \mathrm{s}^{2}\right)$

lacks damping of the spindle vibration. Velocity estimates $\hat{\omega}_{1}, \hat{\omega}_{2}$, and $\hat{\omega}_{3}$ and the corresponding elements of $\xi$ are shown in Fig. 5 and acceleration $\hat{\psi}_{1}, \hat{\psi}_{2}$, and $\hat{\psi}_{3}$ and the corresponding elements of $\xi$ are shown in Fig. 6. The input estimates almost coincide with the measurements, and the residuals on the equations of motion are small. The residuals on the position measurements indicate that there must be some non-modeled dynamics in the system with a significant effect on the end-effector position. It is not difficult to find these in the system: the belt ends are joined together in a pre-stress unit between springs under each slide (Heeren [4]). The slides can easily be displaced over $5 \mathrm{~mm}$ by hand, while the belts are kept in place. This may explain the lower stiffness estimate, because this estimate may represent a global stiffness for serial springs.

From the above results it can be concluded that the relatively simple model structure chosen is capable of describing the measurement data to a considerable extent, using the above parameter values. The model can be improved by adding degrees of freedom for the non-modeled deformations in the system. This will not be done here, because the estimation would be substantially complicated. Moreover, the damping of the flexible spindle could be modeled. However, the estimated model is useful for controller design, 
because a great part of the input required for tracking the desired path can simply be calculated from the model, reducing the feedback effort of the controller.

\section{Conclusions}

An estimation method for nonlinear mechanical systems has been developed that takes into account the a priori knowledge of the system in the form of the equations of motion and the measurement equations. The method determines estimates of the unknown model parametes and of the model variables, i.e., positions, velocities, accelerations and inputs, such as forces and currents. The estimates are optimal, i.e., they minimize the model residuals, given the measurement data.

The optimal estimation method is capable of detecting model errors, because different weights can be applied to the model equations. After the model error has been detected, the corresponding equations can be corrected or ignored, provided that observability is preserved.

It is possible that different choices for $\mathbf{q}_{0}$ result in different local minima of $J$. Thus, the choice of $\mathbf{q}_{0}$ may be crucial, depending on the robustness of the optimal estimation concept, which cannot yet be assessed. The cases studied did not show convergence problems, however, convergence is expected to be a potential problem in some cases. If so, other numerical methods for solving TPBVP's may be suitable for the problem at hand.

In order to verify the practical use of the optimal estimation method an existing mechanical system, i.c. an $x y$-table, has been looked at. The relation between motor currents and end-effector position obtained by the optimal estimation method shows residuals of less than three percent. The elastic deformation of the spindle has been qualitatively well observed. On the basis of the residuals it is expected that a controller based on the estimated model can take into account the inertia, friction and deformation in the system. A residual analysis revealed some non-modeled dynamics in the system. If necessary, the model structure can be improved.

To conclude, it should be noted that the optimal estimation concept described in this paper can probably be applied to a much wider range of systems, for instance, in the field of process control and estimation of material models.

\section{References}

1 Eykhoff, P., System Identification: Parameter and State Estimation, Wiley Interscience, 1974

2 Isidori, A., Lectures on Non-Linear Control, Lecture Notes, University of Rome, 1987.

3 Misawa, E., and Hedrick, J., "Non-linear Observers-A State-of-the-Art Survey," ASME Journal of Dynamic Systems, MEasurement, and ConTROL, Vol. 111, 1989.

4 Heeren, T., "On Control of Manipulators," Ph.D. thesis, Eindhoven University of Technology, 1989.

5 Paul, R., Robot Manipulators, Mathematics, Programming and Control, MIT Press, 1981.

6 Walter, E., Identifiability of State Space Models, Springer-Verlag Berlin Heidelberg, 1982.

7 Tunati, E. and Tarn, T., "New Results for the Identifiability of Non-linear Systems," IEEE Trans. on Automatic Control, Vol. 32, No. 2, 1987.

8 Jacobson, D., and Mayne, D., Differential Dynamic Programming, American Elsevier, 1970.

9 Molengraft, M. J. G. van de, "Identification of Non-linear Mechanical Systems for Control Application," Ph.D. thesis, Eindhoven University of Technology, 1990.

10 MAPLE Reference Manual 5th Edition, WATCOM Publ. Lim., 1988

11 Vincent, H., "Derivation and Identification of the Model of a Mechanical Manipulator," Report WFW 90.022, Eindhoven University of Technology, 1990.
Sufficient Conditions for Monotonic Discrete Time Step Responses ${ }^{1}$

\section{Bernardo León de la Barra ${ }^{2}$}

Simple sufficient conditions for monotonic discrete time step responses are obtained and expressed in terms of the relative locations of discrete time zeros and poles. Frequency domain arguments are also introduced to complement these conditions. Simple examples are used to illustrate the main issues.

\section{Introduction}

Some very recent theoretical contributions have been made on the design of nonovershooting, or overshoot bounded, discrete time control systems. For example, Deodhare and Vidyasagar $(1990,1992)$ have applied infinite linear programming techniques to the design of nonovershooting discrete time control systems when the reference signal is a step. Dahleh and Pearson (1988) studied the more general problem of minimizing the amplitude of a regulated output, due to a specific bounded input, also in a SISO discrete time setting. Moreover, Moore and Bhattacharyya (1990), and Hill and Halpern (1993), considered discrete time controller design for minimal overshoot by making use of additional linear programming techniques. However valuable, these contributions do not offer a clear picture of how and to what extent variations in the relative locations of zeros and poles of plant and compensator may influence the performance index.

An important related problem is the characterization of the discrete time systems which display no relative extrema in the time domain step response. Note that this feature will usually be highly desirable in industrial applications which involve shape or machine tool axis control, where even small deviations from a "monotonic path" can reduce the quality of the final product.

In this paper we provide a simple sufficiency test for monotonic discrete time step responses. This test makes explicit use of the relative locations of zeros and poles of the transfer function under consideration, can be easily carried out by inspection, and includes a reasonably large class of discrete time transfer functions. A novel feature of this work is the use of simple frequency domain arguments which provide additional insight into the nature of the conditions ensuring monotonicity.

\section{Preliminaries}

In this paper we consider an asymptotically stable, proper, discrete time transfer function $G(z)$ having nonzero d.c. gain. We also introduce the following definitions.

\footnotetext{
${ }^{j}$ León de la Barra (1992b) constitutes an earlier version of this work.

${ }^{2}$ Automatic Control Group, Department of Electrical Engineering, University of Chile, PO Box 412-3, Santiago, Chile.

Contributed by the Dynamic Systems and Control Division of the AMERICAN SocietY of Mechanical EngINEERs. Manuscript received by the DSCD September 8, 1992; revised manuscript received September 23, 1993. Associate Technical Editor: $\mathrm{S}$. Jayasuriya.
} 